\title{
Geomagnetic storms and the occurrence of phase slips in the reception of GPS signals
}

\author{
Edward L. Afraimovich, Oleg S. Lesyuta, Igor I. Ushakov and Sergey V. Voeykov \\ Institute of Solar-Terrestrial Physics SD, Russian Academy of Sciences, Irkutsk, Russia
}

\begin{abstract}
We have investigated a dependence of the relative density of GPS phase slips on the geomagnetic disturbance level. The study is based on using Internet-available selected data from the global GPS network, with the simultaneously handled number of receiving stations ranging from 160 to 323 . The analysis used four days from the period 1999-2000, with the values of the geomagnetic field disturbance index $D s t$ from 5 to $-300 \mathrm{nT}$. During strong magnetic storms, the relative density of phase slips on mid-latitudes exceeds that for magnetically quiet days by one-two orders of magnitude as a minimum, and reaches a few percent of the total density of observations. Furthermore, the level of phase slips for the GPS satellites located on the sunward side of the Earth was by a factor of 5-10 larger compared with the opposite side of the Earth. The level of slips of $L_{1}$ phase measurements at the fundamental GPS frequency is at least one order of magnitude lower than that in $L_{1}-L_{2}$ measurements. The slips of $L_{1}-L_{2}$ measurements are most likely to be caused by the high level of slips of $L_{2}$ phase measurements at the auxiliary frequency. As an alternative, we developed and tested a new method for determining TEC variations using only data on the pseudo-range and phase measurements at fundamental frequency $L_{1}$. The standard deviation of the TEC variations which were obtained in phase measurements at two frequencies, $L_{1}-L_{2}$, and at fundamental frequency $L_{1}$, does not exceed $0.1 \mathrm{TECU}$, which permits this method to be used in strong disturbance conditions when phase slips at auxiliary frequency $L_{2}$ are observed.
\end{abstract}

Key words phase slips - geomagnetic disturbances total electron content

\section{Introduction}

The GPS satellite navigation system has become a powerful factor of scientific and technological progress worldwide, and enjoys wide use in a great variety of human activities. In this connection, much attention is given to continuous perfection of the GPS system and to

Mailing address: Prof. Edward L. Afraimovich, Institute of Solar-Terrestrial Physics SD, Russian Academy of Sciences, P.O. Box 4026, Irkutsk, 664033, Russia; e-mail: afra@iszf.irk.ru the widening of the scope of its application for solving the navigation problems themselves, as well as for developing higher-precision systems for time and accuracy determinations. Even greater capabilities are expected in the near future through the combined use of the GPS with a similar Russian system (GLONASS).

Recently, the GPS system has also gained widespread acceptance in research in the field of geodynamics, in the physics of the Earth's atmosphere, ionosphere and plasmasphere, etc. (Davies and Hartmann, 1997; Klobuchar, 1997). Investigations of this kind are not only of purely scientific interest but are also important for perfection of the GPS system itself.

To address these problems, a global network of receiving GPS stations was set up, which consisted, by August 2001, of no fewer than 900 
points, the data from which are placed on the Internet.

Using two-frequency multichannel receivers of the GPS global navigation system, at almost any point on the globe and at any time simultaneously at two coherently-coupled frequencies $f_{1}=1575.42 \mathrm{MHz}$ and $f_{2}=1227.60$ $\mathrm{MHz}$, highly accurate measurements of the group and phase delays are being made along the Line Of Sight (LOS) between the receiver on the ground and the transmitters on-board the GPS system satellites which are in the zone of reception.

These data, converted to values of Total Electron Content (TEC), are of considerable current use in the study of the regular ionosphere and of disturbances of natural and technogenic origins (solar eclipses, flares, earthquakes, volcanoes, strong thunderstorms, auroral heating, nuclear explosions, chemical explosion events, launches of rockets). We do not cite here the relevant references for reasons of space, which account for hundreds of publications to date (Evans, 1977; Davies and Hartmann, 1997; Klobuchar, 1997).

The study of deep, fast variations in TEC caused by a strong scattering of satellite signals from intense small-scale irregularities of the ionospheric $F_{2}$-layer at equatorial and polar latitudes has a special place among ionospheric investigations based on using satellite (including GPS) signals (Aarons, 1982; Yeh and Liu, 1982; Basu et al., 1988; Aarons et al., 1996, 1997; Klobuchar, 1997; Pi et al., 1997; Aarons and Lin, 1999; Bhattacharrya et al., 2000). The interest in this problem as regards the practical implementation is explained by the fact that as a result of such a scattering, the signal undergoes deep amplitude fadings, which leads to a phase slip at the GPS working frequencies (Skone and Jong, 2000).

A limitation of the cited references is the fact that they use, as input data, essentially the values of TEC determined from the phase difference $L_{1}-L_{2}$ (see below). For that reason, fatal phase slips that totally prohibit measurements of continuous TEC variations, are excluded from the sample statistics reported in the cited references.

In this paper we inquire into the question: What are the statistics of fatal phase slips, and how do they depend on the various geophysical factors (the level of geomagnetic disturbance, the latitudinal and diurnal dependencies)?

We used a new technology of a global detector GLOBDET, and relevant software which makes it possible to automate the acquisition, filtering and pretreatment process of the GPS data received via the Internet (Afraimovich, 2000). This technology is being used to detect, on global and regional scales, the ionospheric effects of strong magnetic storms (Afraimovich et al., 2000a, 2001b), solar flares (Afraimovich, 2000; Afraimovich et al., 2001c), solar eclipses (Afraimovich et al., 1998a), launches of rockets (Afraimovich et al., 2000b), earthquakes (Afraimovich et al., 2001a), etc.

The experimental geometry and general information on the data base used are presented in Section 2. The determination of the relative density of phase slips, and the method of processing the data available from the Internet are briefly outlined in Section 3. Section 4 describes the results obtained for magnetically quiet and disturbed conditions. Results are discussed in Section 5.

\section{Experimental geometry and general information on the data base used}

This study is based on using the data from a global network of GPS receiving stations available from the Internet (http://sopac.ucsd.edu). For a number of reasons, slightly differing sets of GPS stations were chosen for the various events under investigation; however, the experimental geometry for all events was virtually identical. The analysis used a set of stations (from 160 to 323 ) with a relatively even distribution across the globe. For reasons of space, we do not give here the station coordinates. This information may be obtained from http://sopac.ucsd.edu/cgi-bin/ dbShowArraySitesMap.cgi?array=ALL\&array_ option=siteList.

The set of stations, which we selected out of the part of the global GPS network available to us, covers rather densely North America and Europe; Asia has much poorer coverage. The number of GPS stations in the Pacific and Atlantic oceans is even smaller. However, such 
A pril 6, 2000
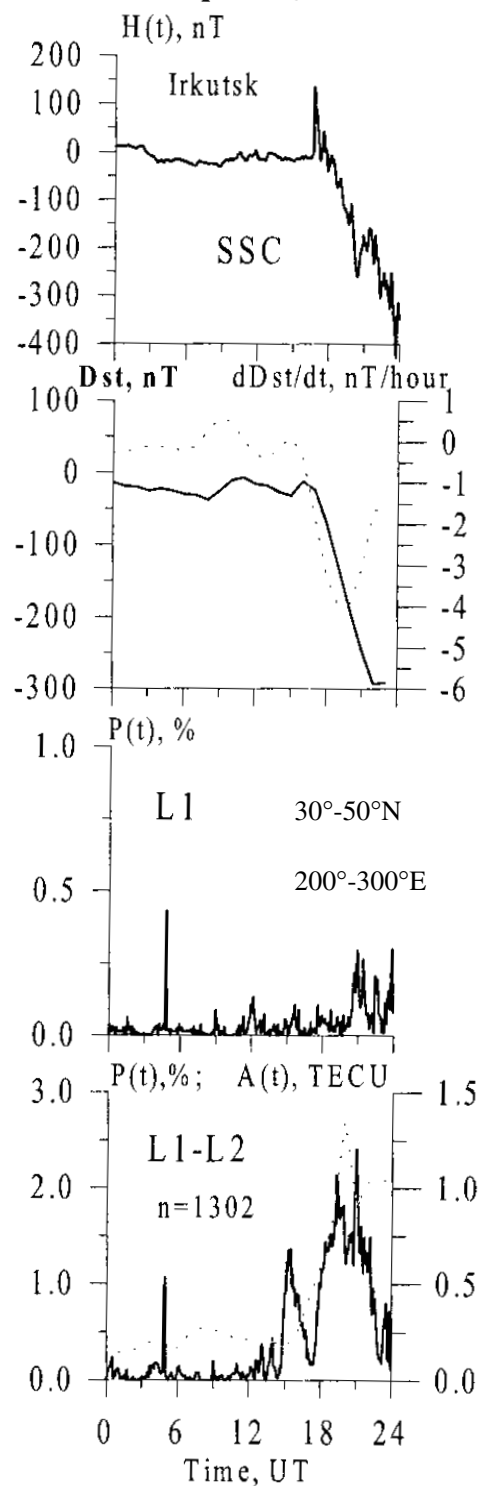

July 15,2000

(a)

(b)

$H(t), n T$
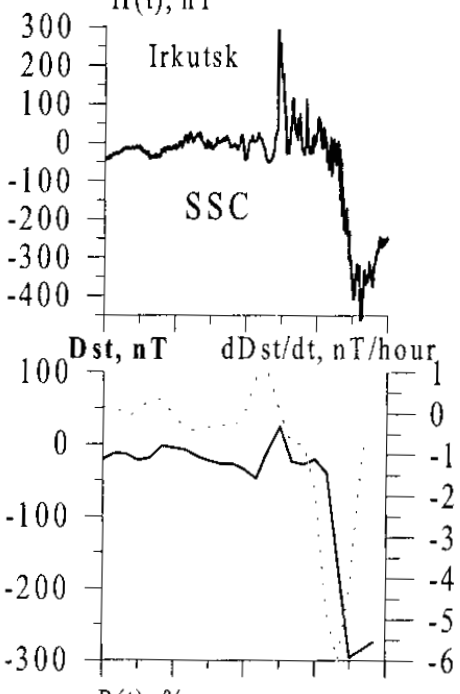

(c)
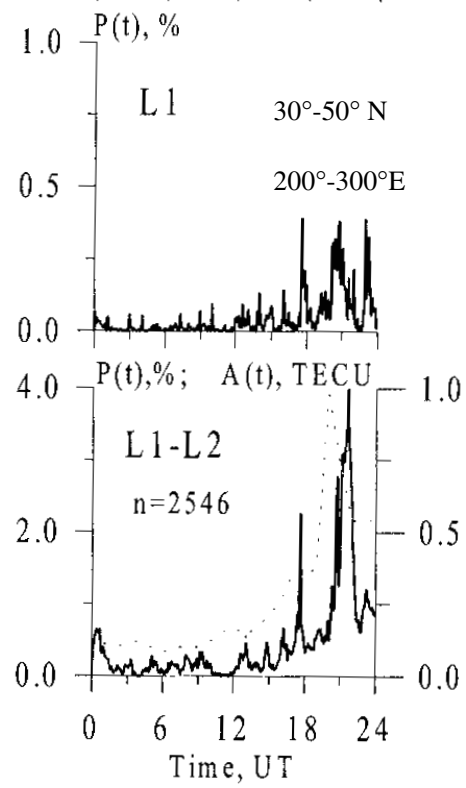

(f)

(e)

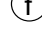

(g)

Fig. 1a-h. Variations of the $H$-component of the geomagnetic field record at station Irkutsk (a); Dst (b), thick line, and the dependence of the time derivative $d(D s t(t)) / d t(\mathrm{~b})$, dashed line, during the magnetic storm of April 6, 2000. The UT-dependence of the relative mean $L_{1}-L_{2}$ slip density $P(t)(\mathrm{d})$, thick line. For comparison, the panel (c) plots the relative mean $L_{1}$ slip density $P(t)$. The same dependencies for a magnetic storm of July 15 , 2000 (e,f,g,h). The SSC times 16:42 UT and 14:37 UT are shown by a vertical bar. For comparison, the dashed line in panels (d) and (h) plots the dependencies $A(t)$ of the TEC variation intensity obtained for the same set of stations as in the case of $P(t)$. 
Edward L. Afraimovich, Oleg S. Lesyuta, Igor I. Ushakov and Sergey V. Voeykov

Table I. Statistics of experiments.

\begin{tabular}{crcccc}
\hline \hline $\mathrm{N}$ & \multicolumn{1}{c}{ Date } & Day number & $\mathrm{m}$ & $D s t_{\max }, \mathrm{nT}$ & $K p_{\max }$ \\
\hline 1 & 29.07 .1999 & 210 & 160 & -40 & 3 \\
2 & 9.01 .2000 & 009 & 323 & -13 & - \\
3 & 6.04 .2000 & 097 & 243 & -293 & 8 \\
4 & 15.07 .2000 & 197 & 306 & -295 & 9 \\
\hline
\end{tabular}

coverage over the globe is already sufficient for a global detection of disturbances with spatial accumulation unavailable before. Thus, in the western hemisphere, the corresponding number of stations can, already today, reach at least 500, and the number of beams to the satellites no fewer than 2000-3000.

The analysis involved four days of the period 1999-2000, with the values of the geomagnetic field disturbance index $D s t$ ranging from 0 to $-300 \mathrm{nT}$ and $K_{p}$ from 2 to 9 . The maximum values of the geomagnetic field disturbance index $D s t_{\max }$ and $K_{p \max }$ are listed in table I.

Figure 1a-h presents the measured variations of the $H$-component of the geomagnetic field at station Irkutsk $\left(52.2^{\circ} \mathrm{N} ; 104.3^{\circ} \mathrm{E}-\mathrm{a}, \mathrm{e}\right)$, and Dst (b,f - thick line) during major magnetic storms on April 6, and July 15, 2000; a correlative analysis of the data is made in Section 4. The SSC times 16:42 UT and 14:37 UT are shown by a vertical bar.

The statistics of the data used in this paper for each of the days under examination are characterized by the information in table I about the number of stations used $m$. The total amount of data exceeds $10^{7} 30$-s observations.

\section{The method of processing the data from the Internet}

The purpose of a preprocessing of the GPS data in this paper is to obtain slip density estimates in measuring the phase difference $L_{1}-L_{2}$, and slips of phase measurement at the fundamental frequency $L_{1}$. Ascertaining the cause of the increase in slip density was also greatly facilitated by estimating the TEC variation intensity for the same stations and time intervals.

To this end, we also obtained normalized distributions of LOS elevations and azimuths, for which slips of measurements of the phase difference $L_{1}-L_{2}$, and phase $L_{1}$ slips were recorded for the time interval of our interest.

The GPS technology provides the means of estimating TEC variations on the basis of phase measurements of TEC I in each of the spaced two-frequency GPS receivers using the formula (Afraimovich et al., 1998a)

$$
\begin{gathered}
I_{0}=\frac{1}{40.308} \frac{f_{1}^{2} f_{2}^{2}}{f_{1}^{2}-f_{2}^{2}}\left[\left(L_{1} \lambda_{1}-L_{2} \lambda_{2}\right)+\right. \\
+ \text { const }+n L]
\end{gathered}
$$

where $L_{1} \lambda_{1}$ and $L_{2} \lambda_{2}$ are phase path increments of the radio signal, caused by the phase delay in the ionosphere (m); $L_{1}$ and $L_{2}$ are the number of full phase rotations, and $\lambda_{1}^{2}$ and $\lambda_{2}$ are the wavelengths $(\mathrm{m})$ for the frequencies $f_{1}$ and $f_{2}$, respectively; const is some unknown initial phase path (m); and $n L$ is the error in determination of the phase path $(\mathrm{m})$.

Phase measurements in the GPS can be made with a high degree of accuracy corresponding to the error of TEC determination of at least $10^{14} \mathrm{~m}^{2}$ when averaged on a 30 -s time interval, with some uncertainty of the initial value of TEC, however (Hofmann-Wellenhof et al., 1992). For definiteness, we bring the TEC variations into the region of positive values with the minimum value equal to 0 by adjusting the constant term const in eq. (3.1). The TECU (Total Electron Content Units), which is equal to $10^{16} \mathrm{~m}^{-2}$ and is commonly accepted in the literature, will be used throughout the text. 
Primary data include series of «oblique» values of TEC $I_{0}(t)$, as well as the corresponding series of elevations $\theta(t)$ and azimuths $\alpha(t)$ along LOS to the satellite calculated using our developed CONVTEC program which converts the GPS system standard RINEX-files on the Internet (Gurtner, 1993). Series of the values of elevations $\theta(t)$ and azimuths $\alpha(t)$ of the beam to the satellite were used to determine the coordinates of subionospheric points, and to convert the «oblique» TEC $I_{0}(t)$ to the corresponding value of the «vertical» TEC $I(t)$ by employing the technique reported by Klobuchar (1986).

\subsection{The relative density of difference phase $L_{1}-L_{2}$ slips and of phase $L_{1}$ slips}

We hold fixed a slip of the phase difference $L_{1}-L_{2}$ in the case where the modulus of the TEC increment for a time interval of $30 \mathrm{~s}$ (which is a standard one for the GPS data placed on the Internet), exceeds the specified threshold of order, for example, 100-200 TECU. A slip of phase $L_{1}$ is also fixed in a similar manner but with a much larger threshold and with due regard for the time varying distance to the satellite.

Thus we point out that it is the fatal slips which totally prohibit the determination of the increment of TEC from the measures value of the phase difference $L_{1}-L_{2}$. At the same time there can be numerous phase slips whose absolute value is smaller than the threshold which we selected. Such slips that accompany TEC variations of only a few TECU, are caused by ionospheric irregularity effects, and have been thoroughly studied in a large number of publications (Basu et al., 1988; Aarons et al., 1996, 1997; Klobuchar, 1997; Pi et al., 1997; Aarons and Lin, 1999; Bhattacharrya et al., 2000).

As a result of a pretreatment of the RINEXfiles, we have the number $S$ of phase slips within a single selected time interval $d T=5 \mathrm{~min}$, as well as the corresponding number $M$ of observations required for normalizing the data. These data for each of the GPS satellites were then averaged for all the stations selected in order to infer the mean density of observations $M(t)$ and the mean density of phase slips $S(t)$. In the middle of the observed satellite pass, the density of observations $M(t)$ averages $10 \pm 1$ (30-s counts); at the beginning and end of the pass it can decrease because the time intervals of observation of a given satellite at elevations larger than that specified do not coincide at different stations. Subsequently, we calculated the mean relative density of phase slips $P(t)=$ $S(t) / M(t), \%$. Furthermore, the daily mean value of the relative number of phase slips $\langle P\rangle$ that was averaged over all GPS satellites and stations was useful for our analysis.

As would be expected the mean observation density $M(t)$ for a single satellite exhibits a diurnal variation that is determined by the satellite's orbit, and varies over the range from 0 to 8 .

\subsection{Estimation of the TEC variation intensity}

We used the series $I(t)$, containing neither slips of the phase difference $L_{1}-L_{2}$ nor gaps of counts, to estimate the TEC variation intensity for the same sets of stations and time intervals as used in estimating the phase slip density.

To exclude the variations of the regular ionosphere, as well as trends introduced by the motion of the satellite, we employ the procedure of removing the linear trend by preliminarily smoothing the initial series with a selected time window of a duration of about $60 \mathrm{~min}$. In a subsequent treatment, we use the standard deviation of the TEC variations $d I(t)$, thus filtered, as an estimate of the TEC variation intensity $A$ (see Section 4).

Figure 2a gives an example of a typical weakly disturbed variation in «vertical» TEC $I(t)$ for station WES2 $\left(42.6^{\circ} \mathrm{N}, 288.5^{\circ} \mathrm{E}\right)$; satellite number PRN10 on July 15, 2000 for the time interval 14:00-16:00 UT, preceding the onset of a geomagnetic disturbance near the WES2 station. For this same series, fig. $2 b$ presents the $d I(t)$ variations that were filtered out from the $I(t)$ series ( $\mathrm{rms}$ of $d I(t)$ is smaller then 0.2 TECU). In this paper, we use the term PRN (Pseudo Random Noise) to designate the satellite number (Hofmann-Wellenhof et al., 1992). 

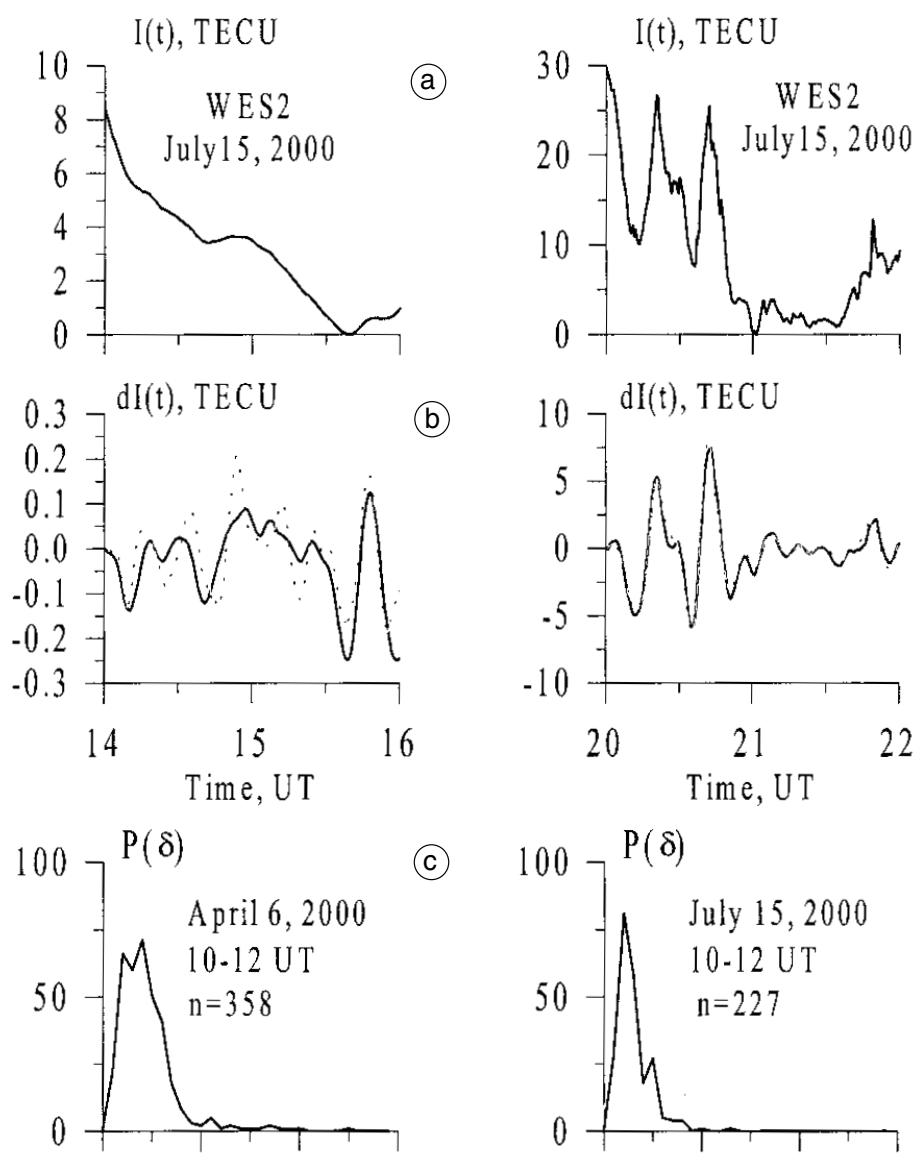

(C)
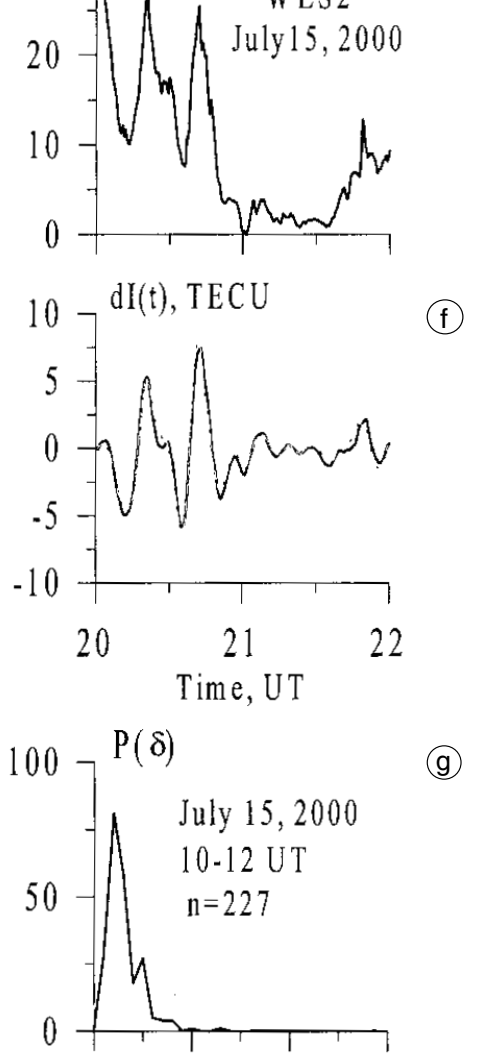

(f)
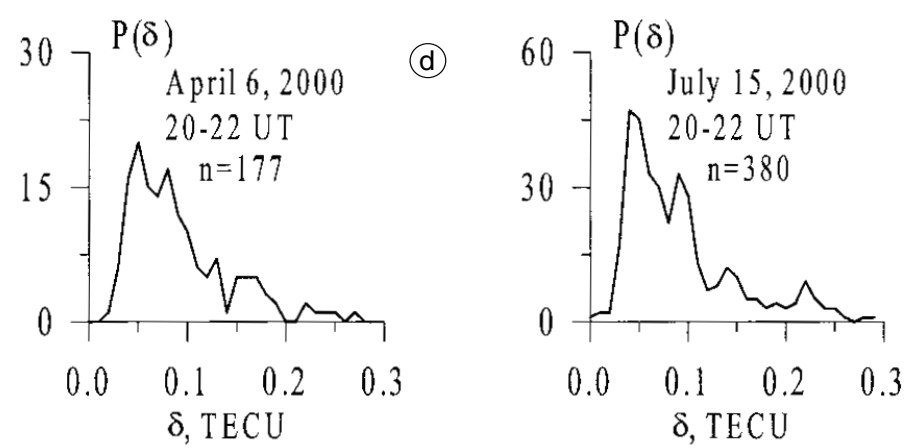

(9)

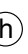

Fig. 2a-h. Time dependencies of the «vertical» TEC $I(t)$, measured at station WES2 (a) prior to the onset of the geomagnetic disturbance of July 15, 2000, and at station WES2 thereafter (e). The $d I(t)$ variations filtered from the $I(t)$ series by removing the trend with a 60 -min window for this station (b,f). For comparison, the dashed line in panels (b) and (f) plots the $d I(t)$ variations calculated from values of the pseudo-range $C_{1}$ and of the phase $L_{1}$. Distributions $P(\delta)$ of the standard deviation $\delta$ of the TEC variations obtained in phase measurements at two frequencies $L_{1}-L_{2}$ and at the $L_{1}$ only (c,d,g,h). 
Strong variations in TEC variation intensity occurred near station WES2 literally within $6 \mathrm{~h}$. Figure 2e and fig. 2f present the dependencies $I(t)$ and $d I(t)$ for station WES2 for the time interval 20:00-22:00 UT (PRN23). As is evident from the figure, the TEC variations increased in intensity by a factor of at least 30 when compared with the time interval 14:00-16:00 UT (fig. 2a,b).

\subsection{Conditions and limitations of data processing}

Slips of phase measurements can be caused by reception conditions for the signal in the neighbourhood of the receiver (interference from thunderstorms, radiointerferences), which is particularly pronounced at low elevations $\theta$. To exclude the influence of the signal reception conditions, in this paper we used only observations with satellite elevations $\theta$ larger than $30^{\circ}$.

Since we are using a global averaging of the number of phase slips for all LOS and stations, as a consequence of the uneven distribution of stations the proportion of midlatitude stations of North America and, to a lesser extent, of Europe is predominant (see above). At the same time, the number of stations in the polar region of the Northern Hemisphere and in the equatorial zone was found to be quite sufficient for a comparative analysis. To compare the results, we chose 3 latitude ranges: high latitudes $50-80^{\circ} \mathrm{N}$; mid-latitudes $30-50^{\circ} \mathrm{N}$; and equatorial zone $30^{\circ} \mathrm{S}-30^{\circ} \mathrm{N}$.

We selected also the data according to the types of two-frequency receivers, with which the GPS global network sites are equipped (the relevant information is contained in the initial RINEX format).

\section{Results derived from analyzing the relative density of phase slips}

\subsection{Magnetically quiet days}

Figure 3a-f plots the local time LTdependence of the relative mean slip density $P(t)$ obtained by averaging the data from all satellites in the latitude range $0-360^{\circ} \mathrm{E}$ irrespective of the type of GPS receivers for the magnetically quiet days of July 29, 1999 (at the left) and January 9, 2000 (at the right). The local time for each GPS station was calculated, based on the value of its geographic longitude. The number $n$ of satellite passes used to carry out an averaging is marked in all panels.

As is evident from fig. 3b, the phase slips on a magnetically quiet day at mid-latitudes have a sporadic character. The daily mean value of the relative density of phase slips $\langle P\rangle$, averaged over all GPS satellites and stations, was $0.017 \%$ for the magnetically quiet day of July 29, 1999. Similar data were also obtained for high latitudes (fig. 3a).

In the equatorial zone, however, even on a magnetically quiet day, the density of phase slips exceeds the latitudinally mean value of $P(t)$ at least by a factor of 15 , and shows a strongly pronounced LT-dependence, with a maximum value of $1.52 \%$ (fig. 3c).

For the other magnetically quiet day of January 9, 2000, however, the mean value of $\langle P\rangle$ at mid-latitudes was already larger $0.06 \%$ (fig. 3e). For the diurnal $P(t)$-dependence on January 9, 2000, one can point out the irregularity of the mean density of phase slips as a function of local time LT.

\subsection{Magnetic storms of April 6 and July 15, 2000}

A totally different picture was observed on April 6, 2000 during a strong magnetic storm with a well-defined SSC.

Figure 1d (thick line) presents the variations of the UT-dependence of the relative mean slip density $P(t)$ obtained for the territory of North America $\left(200-300^{\circ} \mathrm{E}\right)$ at mid-latitudes $30-50^{\circ} \mathrm{N}$ by averaging the data from all satellites. In this case, for the purpose of achieving a clearer detection of the effect of the magnetic storm SSC influence on the $P(t)$-dependence, we chose only those GPS stations which were on the dayside of the Earth at the SSC time (North America region). Noteworthy is a well-defined effect of an increase in the density of phase slips that occurred after SSC. 
July 29,1999

January 9,2000

\section{$0-360^{\circ} \mathrm{E}$}

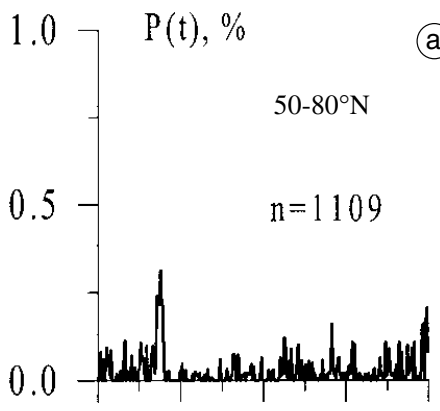

(a) $\quad 1.07 \mathrm{P}(\mathrm{t}), \%$

(d)
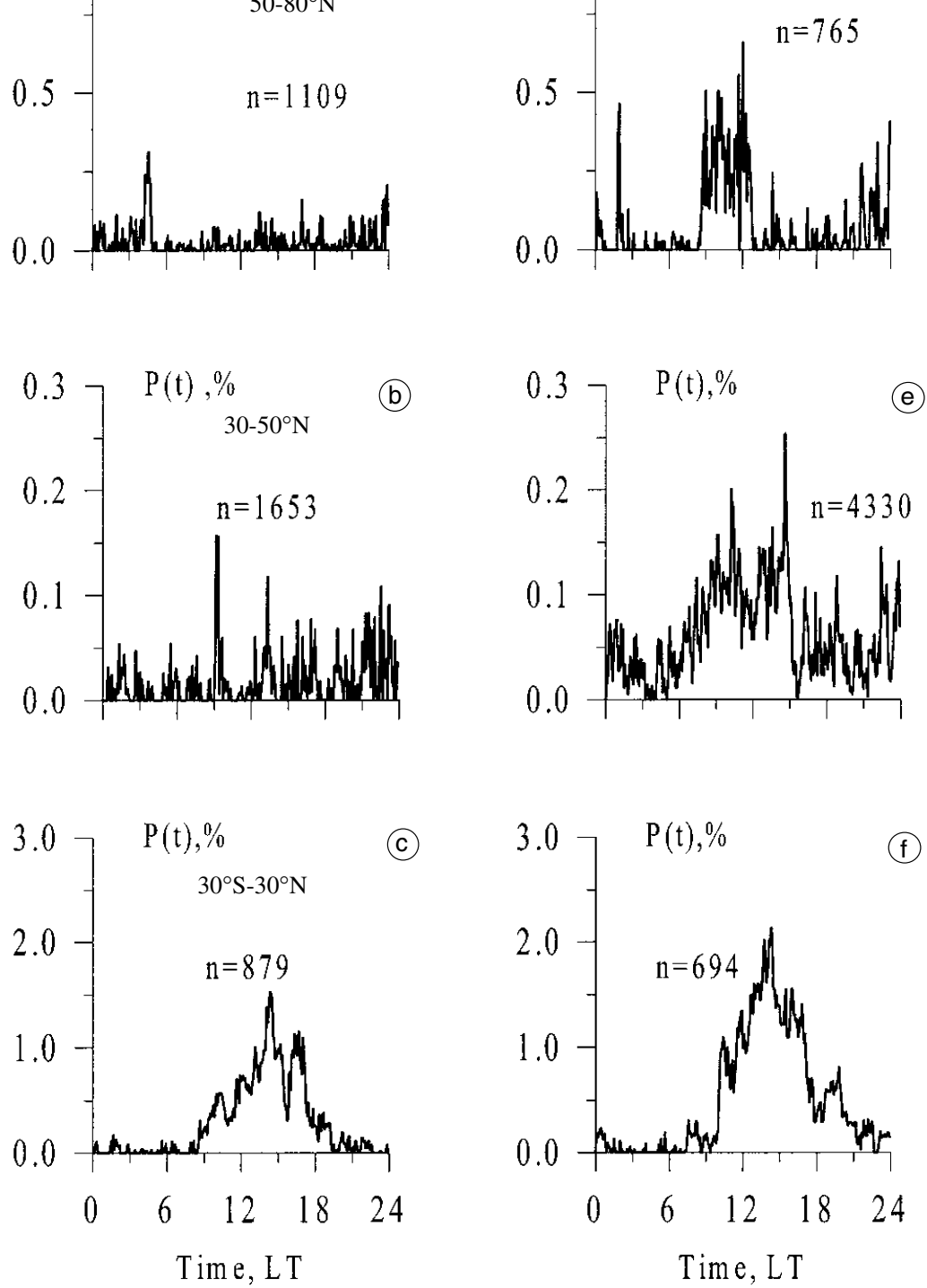

Fig. 3a-f. Local time LT-dependence of the relative mean slip density $P(t)$, obtained by averaging the data from all GPS satellites in the longitude range $0-360^{\circ} \mathrm{E}$ irrespective of the type of GPS receivers for the magnetically quiet days of July 29, 1999 (at the left), and January 9, 2000 (at the right): a,d) high latitudes 50-80 N; b,e) midlatitudes $30-50^{\circ} \mathrm{N}$, and $\mathrm{c}, \mathrm{f}$ ) equatorial zone $30^{\circ} \mathrm{S}-30^{\circ} \mathrm{N}$. 
A maximum mean slip density $P_{\max }=2.4 \%$ is attained 3-4 h after an SSC. The same values, averaged over all observed satellites and midlatitude stations $\left(0-360^{\circ} \mathrm{E}\right)$ but as a function of local time LT, are plotted in fig. 4b.

First of all, it should be noted that the relative density of phase slips $P(t)$ exceeds that for magnetically quiet days by one (when compared with January 9, 2000) or even two (when compared with July 29, 1999) orders of magnitude, and reaches a few percent of the total observation density. The mean value of $\langle P\rangle$ for this storm is $0.67 \%$, which is by a factor of 40 larger than that of $\langle P\rangle$ for July 29,1999 , and by a factor of 10 larger than that for January 9, 2000.

It was also found that the averaged (over all satellites) level of phase slips for the GPS satellites on the subsolar side of the Earth is by a factor of 10 larger than that on the opposite side of the Earth (fig. 4b).

Similar dependencies with a maximum slip density $P_{\max }=3.37 \%$ and a sharply pronounced diurnal dependence were also obtained for equatorial latitudes (fig. 4c). On the other hand, although the high latitudes show a 10-fold increase of $\langle P\rangle$ as against a magnetically quiet day, no LTdependence is observed (fig. 4a).

A similar result confirming all of the abovementioned features of the April 6, 2000 storm was also obtained for the other magnetic storm of July 15, 2000 (see the measurements at magnetic observatory Irkutsk in fig. 1e, the Universal Time (UT) dependence in fig. $1 \mathrm{~h}$ and the Local Time (LT) dependencies of the relative mean density of phase slips $P(t)$ obtained by averaging the data from all GPS satellites, in fig. 4d-f.

The mean value of $\langle P\rangle$ for this storm at midlatitudes is $0.34 \%$, which is also in appreciable excess of the level of phase slips for magnetically quiet days. The effect of an increase in the density of phase slips after SSC is clearly pronounced for this storm as well (fig. 1h, thick line; see below).

\subsection{Correlation of the increase in slip density and TEC variation intensity}

Equatorial latitudes are characterized by strong scintillations of the transionospheric signal caused by the scattering from $F_{2}$-region ionization irregularities (Aarons, 1982; Yeh and Liu, 1982; Basu et al., 1988; Aarons et al., 1996, 1997; Klobuchar, 1997; Pi et al., 1997; Aarons and Lin, 1999; Bhattacharrya et al., 2000).

Since during the active phase of the magnetic storm the mid-latitude ionosphere becomes increasingly inhomogeneous, it might be anticipated that a similar mechanism is able to cause appreciable scintillations of the GPS signal at mid-latitudes as well. To verify this hypothesis, we determined the dependencies $A(t)$ of the TEC variation intensity obtained for the same set of stations as in the case of $P(t)$ (see Section 3 ).

The dependencies $A(t)$ as a function of UT (fig. 1d,h - dashed line) and LT (fig. 4b,e - dashed line) are presented below by averaging (over all GPS satellites and stations) the standard deviation of the variations $d I(t)$ (Afraimovich et al., 2001b).

In fig. $1 \mathrm{~h}$, the dashed line represents the dependence $A(t)$ of the TEC variation intensity obtained for all satellites and for the territory of North America at mid-latitudes $30-50^{\circ} \mathrm{N}$ during the magnetic storm of July 15, 2000. As is apparent from this figure, the dependence $A(t)$ correlates quite well with the UT-dependence of the relative mean slip density $P(t)$ calculated from the same set of stations as in the case of $A(t)$.

A similar result on the UT-dependence was also obtained for a major magnetic storm of April 6, 2000 (fig. 1d, dashed line). A correlation of the increase in slip density and in TEC variation intensity is shown as clearly by the LT-dependencies $A(t)$ for the magnetic storms of April 6 and July 15, 2000, presented in fig. 4b, e (dashed line).

It was found that an increase in the level of geomagnetic activity is accompanied by an increase in total intensity of $A(t)$; however, it correlates not with the absolute level of $D s t$, but with the value of the time derivative of Dst (a maximum correlation coefficient reaches -0.94 , fig. 1b,f, dashed line). The derivative $d(D s t) / d t$ was obtained from the dependence $\operatorname{Dst}(t)$ (fig. $1 \mathrm{~b}, \mathrm{f}$, dashed line) that was smoothed with a 7-h time window. This result is in reasonably good agreement with the conclusions drawn by Ho et al. (1998) and Afraimovich et al. (2000a, 2001b). 
A pril 6, 2000

$30-50^{\circ} \mathrm{N} ; 0-360^{\circ} \mathrm{E}$

July 15,2000

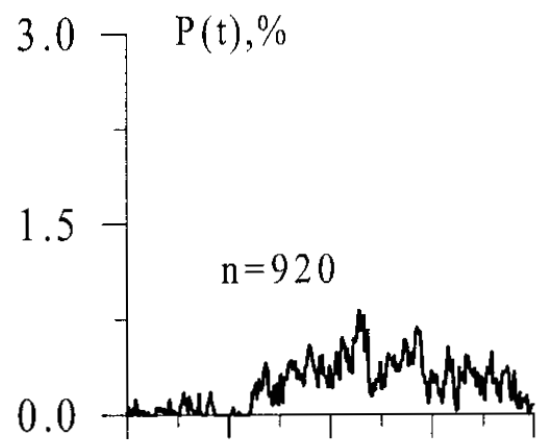

(a)

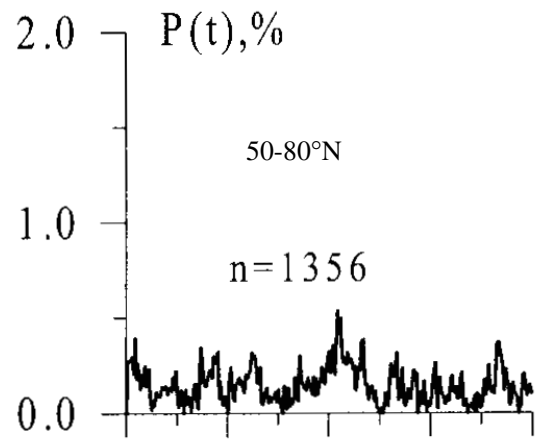

(b)
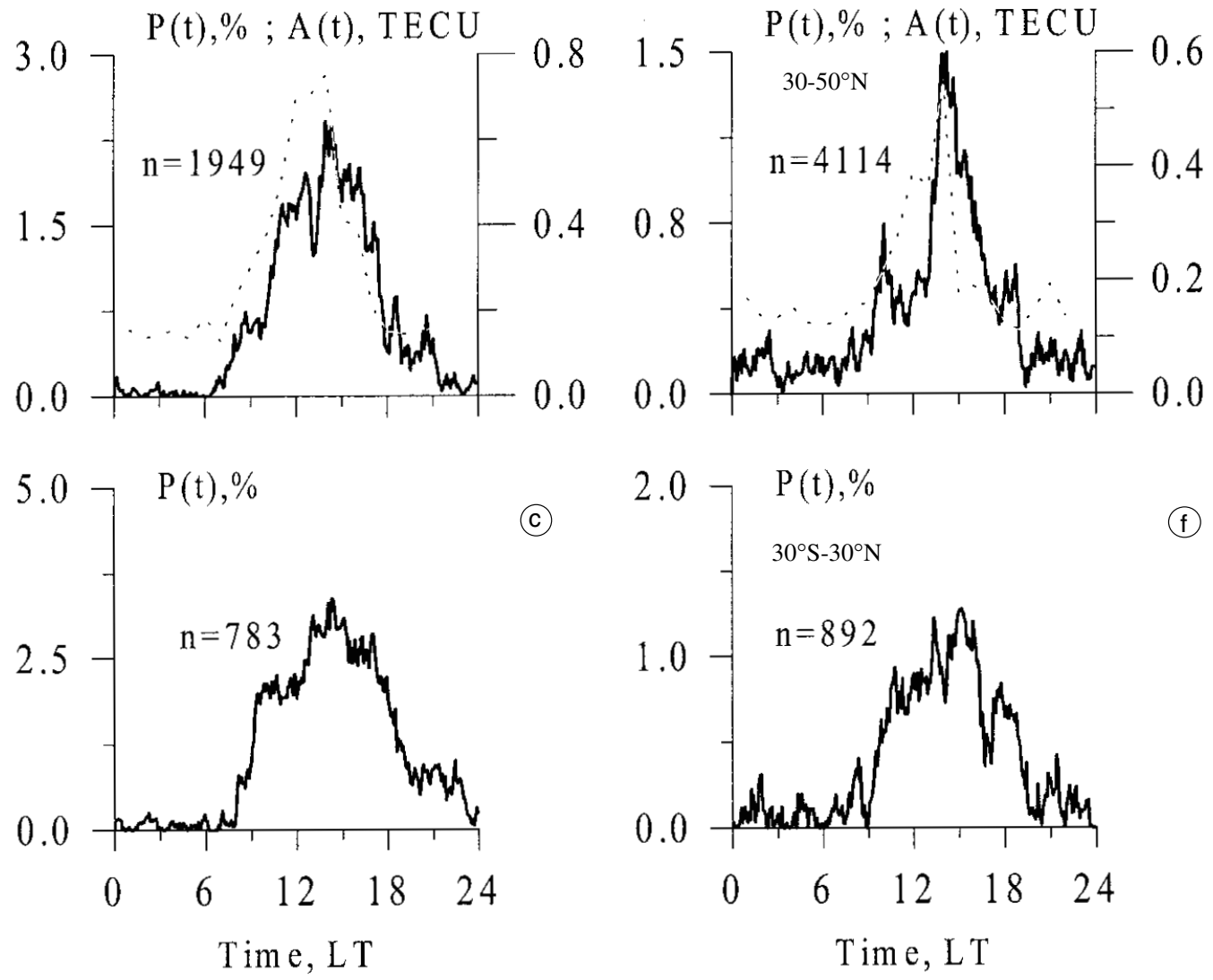

Fig. 4a-f. Same as in fig. 3a-f, but for the magnetic storms on April 6 (at the left) and July 15, 2000 (at the right) at mid latitudes. For comparison, the dashed line in panels (b) and (e) plots the dependencies $A(t)$ of the TEC variation intensity obtained for the same set of stations as in the case of $P(t)$. 


\subsection{The dependence of the slip density of phase measurements $L_{1}-L_{2}$ and $L_{1}$ on the type of GPS receivers}

We carried out an analysis of the dependence of the density of fatal slips on the type of GPS receivers; furthermore, we verified slips for which of the channels $\left(L_{1}\right.$ or $\left.L_{2}\right)$ are the cause of slips in measurements of the phase difference $L_{1}-L_{2}$.

The sample statistics of phase slips for the main types of two-frequency receivers (ASHTECH, TRIMBLE, AOA), installed at the global GPS network sites, are presented in fig. 5a-f and fig. 6a-f.

An analysis of slips in measuring the phase difference $L_{1}-L_{2}$ and of the phase $L_{1}$ was carried out for the magnetic storms of April 6, and July $15,2000$.

Figure 5a-f plots the UT-dependencies of the relative mean slip density $P(t)$ of phase measurements of $L_{1}-L_{2}$ (at the left) and phase measurements of $L_{1}$ only (at the right) obtained by averaging the data from all satellites in the longitude range $200-300^{\circ} \mathrm{E}$ at the mid-latitudes $30-50^{\circ} \mathrm{N}$ for major magnetic storm on April 6, 2000.

The data from fig. 5a suggest that for the ASHTECH receivers, the slip density of phase measurements at two frequencies $L_{1}-L_{2}$ is by a factor of 5-20 smaller than that for the other types of receivers. These estimates are only slightly exceeded by the slip density for the TRIMBLE receivers (fig. 5b). The AOA receivers are the most susceptible to slips of $L_{1}-L_{2}$ measurements (fig. $5 \mathrm{c}$ ). The mean and maximum values of $P(t)$ exceed the respective values for the ASHTECH receivers during the magnetic storm of April 6, 2000, at least by a factor of 10-20.

Figure 6a-f plots the UT-dependencies of the relative mean slip density $P(t)$ of phase measurements of $L_{1}-L_{2}$ (at the left) and phase measurements of $L_{1}$ only (at the right) obtained by averaging the data from all satellites in the longitude range $0-360^{\circ} \mathrm{E}$ at the equatorial zone $30^{\circ} \mathrm{S}-30^{\circ} \mathrm{N}$ for major magnetic storm on April 6, 2000.

The statistics of the $L_{1}-L_{2}$ slips for the AOA and TRIMBLE receivers differ almost not at all from the data for mid-latitudes. For the ASHTECH receivers, however, the density of slips in the equatorial zone was found to be slightly lower when compared with the AOA receivers.

On the other hand, the level of slips of $L_{1}$ phase measurements at the fundamental GPS frequency (fig. 1c,g, fig. 5 a-f and fig. 6a-f, at the right) is at least one order of magnitude lower than that in $L_{1}-L_{2}$ measurements. The corresponding dependencies $P(t)$ as a function of Universal Time (UT) on the dayside for the magnetic storms of April 6 and July 15, 2000, are plotted in fig. 1c and $\mathrm{g}$, respectively. Although the level of the slips is lower by one order of magnitude (as compared to $P(t)$ for $L_{1}-L_{2}$, fig. $\left.1 \mathrm{~d}, \mathrm{~h}\right)$, the fundamental frequency $L_{1}$ does show an increase in the relative density of slips in the main phase of the magnetic storm.

Similar results of a comparison of the level of $L_{1}-L_{2}$ and $L_{1}$ slips for different types of GPS receivers were also obtained for the magnetic storm of July 15, 2000.

This leads us to conjecture that the slips of $L_{1}-L_{2}$ measurements are most likely to be caused by the high level of slips of $L_{2}$ phase measurements at the auxiliary frequency. According to our data, these slips are observed at equatorial latitudes under quiet conditions as well, and at mid-latitudes they increase with increasing geomagnetic activity.

This means that relatively long-term (about 10 years) data from the global GPS network equipped with several hundred older-generation commercial receivers cannot be exploited in full measure to obtain data on TEC variations, based on a standard processing of the phase difference $L_{1}-L_{2}$. This limitation is legitimate for the period of the main phase of the magnetic storm and of the dayside ionosphere which is characterized by fast, profound TEC variations.

\subsection{Determination of TEC variations at the fundamental frequency $L_{1}$}

Some way out of the situation would be by exploiting the method (Afraimovich et al., 1998b) for determining TEC variations using 
April 6, 2000

\section{$30-50{ }^{\circ} \mathrm{N} ; 200-300^{\circ} \mathrm{E}$}

L $1-\mathrm{L} 2$

L 1
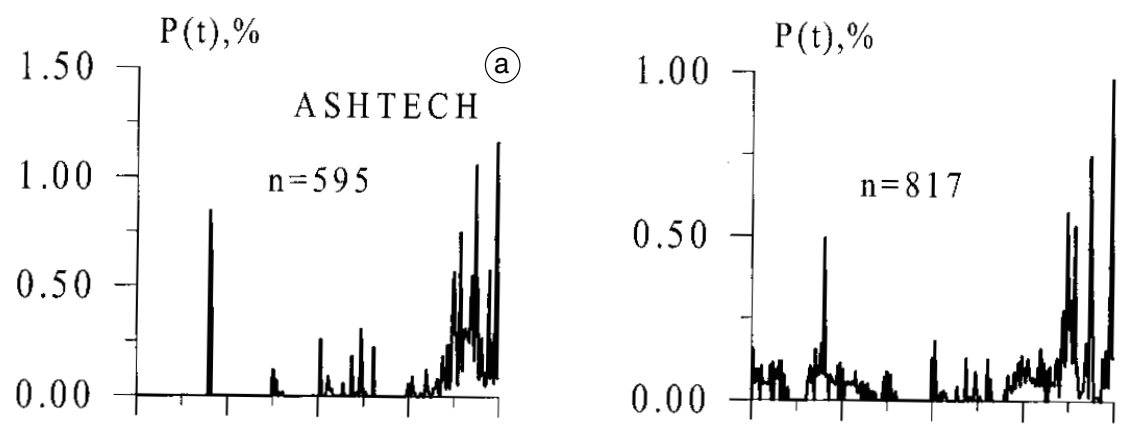

(d)
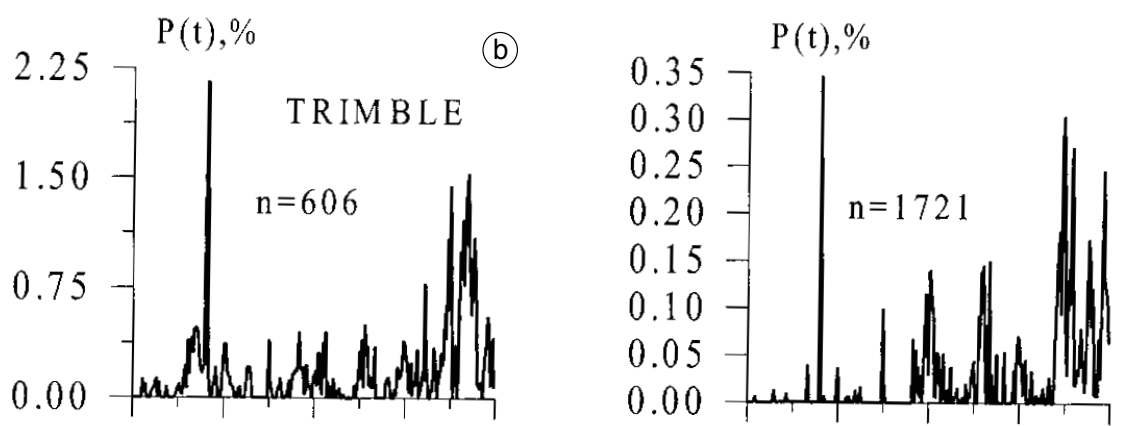

(e)
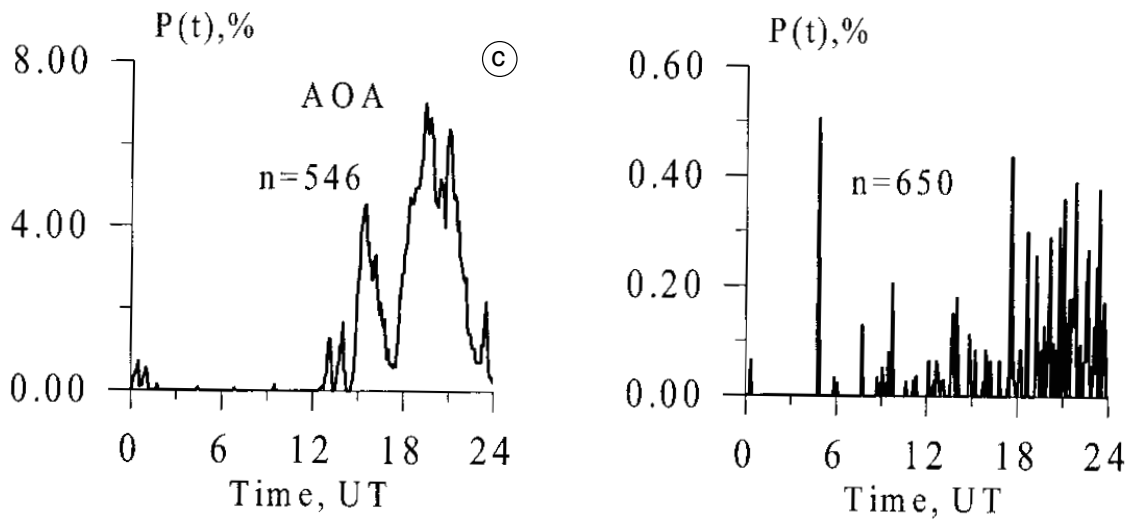

(f)

Fig. 5a-f. UT-dependencies of the relative mean slip density $P(t)$ of $L_{1}-L_{2}$ phase measurements (at the left) and phase measurements of $L_{1}$ only (at the right) for a major magnetic storm of April 6, 2000 (a,d - ASHTECH; $\mathrm{b}, \mathrm{e}$ - TRIMBLE; and c,f - AOA receivers). 


\section{April 6, 2000}

$30^{\circ} \mathrm{S}-30^{\circ} \mathrm{N} ; 0^{\circ}-360^{\circ} \mathrm{E}$

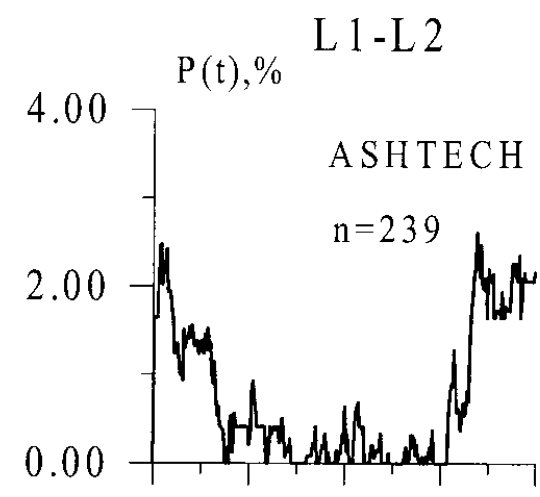

(a)
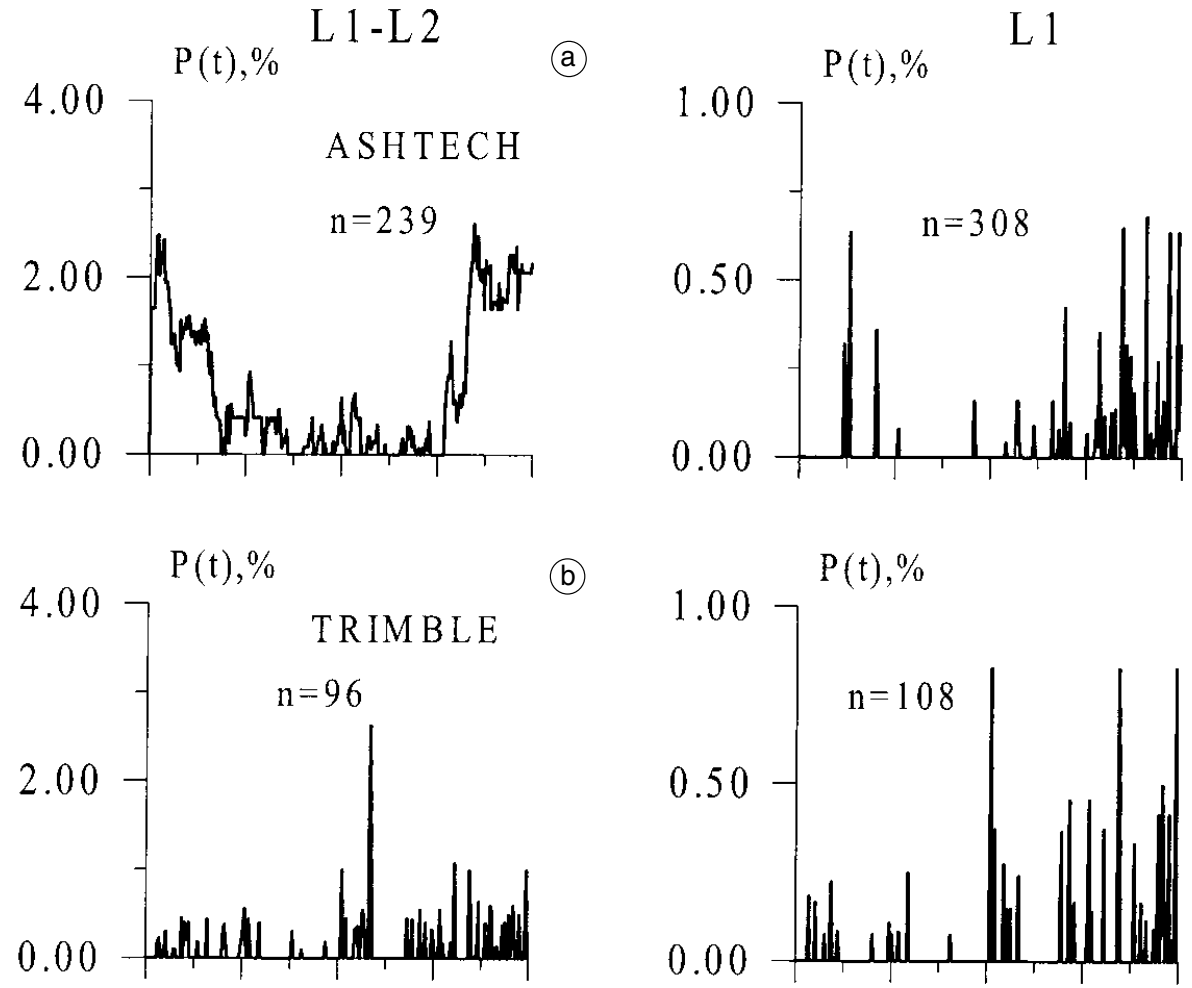

(b)

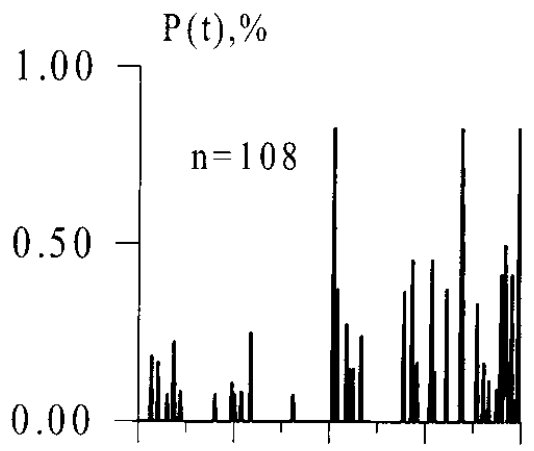

(d)
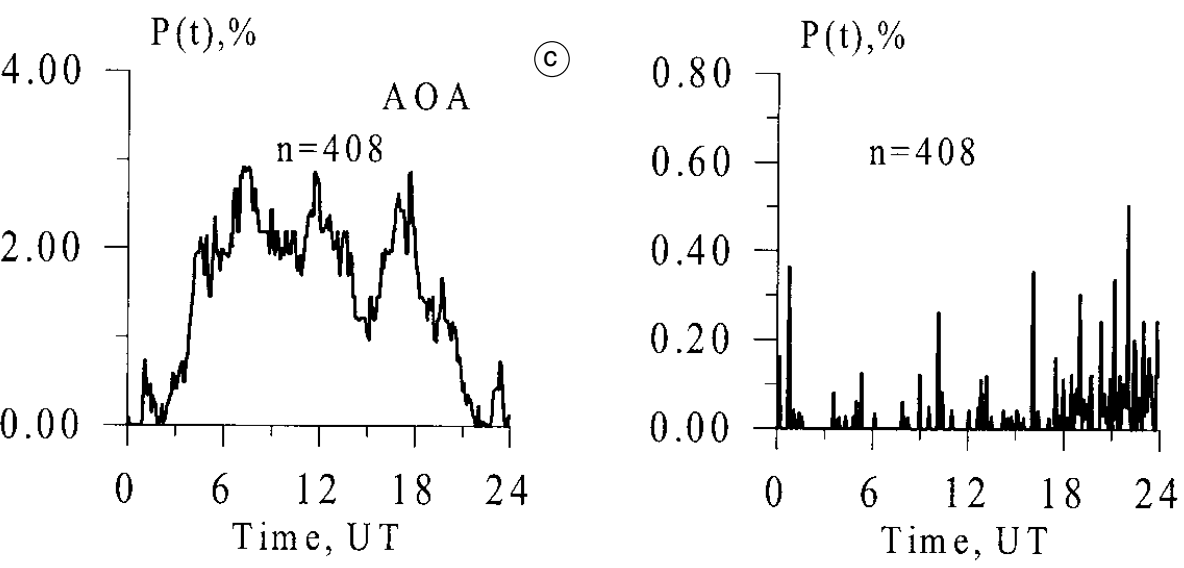

(f)

Fig. 6a-f. Same as in fig. 5a-f, but at the equatorial zone $30^{\circ} \mathrm{S}-30^{\circ} \mathrm{N}$. 
only the data on the pseudo-range $C$ and phase measurements at the fundamental frequency $L_{1}$. In this study the concerned method was tested with the database mentioned in table I.

For testing purposes, time intervals were chosen with no data gaps and slips of phase slip measurements of both $L_{1}-L_{2}$ and $L_{1}$.

Phase changes at frequencies $f_{1}$ and $f_{2}$ may be represented as the sum of different components $\phi_{\Sigma}=\phi_{s}+\phi+\phi_{0}$, where $\phi_{s}=2 \pi \mathrm{C} / \lambda$ is the fundamental component caused by a change of the distance $D$ to the satellite it follows its orbit; $\phi=8.42 \cdot 10^{-7} \mathrm{I} / \mathrm{f}$ is the ionospheric component proportional to TEC at LOS between the receiver and the satellite (Spoelstra and Kelder, 1984); and $\phi_{0}$ is the initial phase. To subtract $\phi_{s}$, it is possible to use current information on the pseudorange $C$ for each satellite (HofmannWellenhof et al., 1992).

For the sake of comparison with the $d I(t)$ variations obtained using the standard TEC measurement technique described in Section 3, from data on the phase difference $L_{1}-L_{2}$ (fig. $2 \mathrm{~b}$, thick lines), dashed lines plot the variations of the "vertical» TEC value obtained by measuring the $L_{1}$ phase and filtered off (as is done for the data on $L_{1}-L_{2}$ ) by removing the trend with the 60-min time window. It is evident from the figure that the difference of the $d I(t)$ dependencies from each other does not exceed in magnitude the value 0.1 TECU, and is more pronounced only for the quiet period 14:0016:00 UT.

For the disturbed time interval 20:00-22:00 UT (fig. 2f), the corresponding dependencies are virtually similar and are not distinguishable. Figure 2a-f also presents the distributions $P(\delta)$ of the standard deviation $\delta$ of TEC variations obtained in phase measurements at two frequencies $L_{1}-L_{2}$ and at the fundamental frequency $L_{1}$. Panels c, d, g and h show the time intervals and the number $n$ of satellite passes, over which the averaging was performed.

During the quiet period 10:00-12:00 UT on April 6 and July 15, 2000 (fig. 2c,g), the most probable value of $\delta$ was 0.04 and 0.05 TECU, and was nearly twice as large as the corresponding value for the same time interval on the magnetically quiet day of July 29, 1999. Furthermore, the mean values of $\delta$ were by a factor of 1.2-1.5 larger than the most probable ones. For the disturbed period 20:00-22:00 UT (fig. 2d,h), the most probable value of $\delta$ remained virtually unchanged; however, the mean values of $\delta$ now were larger than the most probable ones by a factor of 2-3. This is indicative of a more significant discrepancy between results of a calculation of the $d I(t)$ variations from $L_{1}-L_{2}$ and $L_{1}$.

Thus the standard deviation of the TEC variations obtained in phase measurements at two frequencies $L_{1}-L_{2}$ and at the fundamental frequency $L_{1}$ does not exceed 0.1 TECU, which makes this method useful for strong disturbance conditions where slips at the auxiliary frequency $L_{2}$ are observed. Of course, this method is appropriate for solving only those problems, for which it will suffice to single out the TEC variations, and is unsuitable for calculating the absolute value of TEC.

\section{Discussion and conclusions}

The main results of this study may be summarized as follows:

1) We have detected a dependence of the relative density of phase slips in some GPS receivers on the disturbance level of the Earth's magnetosphere during major magnetic storms.

2) During major magnetic storms the relative density of phase slips at mid-latitudes exceeds that for magnetically quiet days at least by one or two orders of magnitude, and reaches a few percent of the total observation density.

3) The level of phase slips for the GPS satellites on the sunward side of the Earth is by a factor of 5-10 times that on the opposite side of the Earth.

4) The level of slips of $L_{1}$ phase measurements at the fundamental GPS frequency is at least one order of magnitude lower than that in $L_{1}-L_{2}$ measurements. The slips of $L_{1}-L_{2}$ measurements are most likely to be caused by the high level of slips of $L_{2}$ phase measurements at the auxiliary frequency.

However, the reason for the slips themselves can include several factors: the influence of additive interferences in the case of a low signal/ noise ratio, the signal scattering from electron 
density irregularities, and the inadequate response of GPS receivers of some types to fast changes in daytime TEC at the auxiliary frequency $L_{2}$. Let us analyze these factors separately.

5) The lower signal/noise ratio at $L_{2}$ is primarily due to the fact that the $L_{2}$ power at the GPS satellite transmitter output is on $6 \mathrm{~dB}$ of magnitude smaller compared with the fundamental frequency $f_{1}$ using C/A code (ICD200, 1994; Langley, 1998). Similar correlations of the effective radiated power of $L_{1}$ (30 watt) and $L_{2}$ (21 watt) signals are also characteristic for the Russian GLONASS system (Kharisov et al., 1998).

Phase slips at $L_{2}$ can also be caused by the lower signal/noise ratio when use is made of commercial noncoded receivers for the frequency $L_{2}$, with which the global GPS network stations are equipped. These receivers have no access to the military $« Y »$ code, and have to use the noncoded or semi-noncoded mode of reception. As a consequence, the signal/noise ratio at the frequency $L_{2}$ is, at best, $13 \mathrm{~dB}$ lower than the mode of fully coded reception.

Thus the difference in signal powers at $L_{1}$ and $L_{2}$ for commercial receivers can become larger than $10 \mathrm{~dB}$, which can lead to an increase in the level of $L_{2}$ slips because of the influence of additive interferences. Different types of GPS receivers respond to this differently; on the whole, however, the picture of the dependence on the local time, latitude range, and on the level of geomagnetic activity remains sufficiently stable.

6) However, the deterioration of the signal/ noise ratio with an increase in the level of geomagnetic disturbance is possible if this is accompanied by an enhancement of the proportion of the signal scattered from ionospheric electron density irregularities. This also involves an increase in the number of phase slips; for a more powerful $L_{1}$ signal, however, the density of slips is an order of magnitude smaller than that for the less powerful $L_{2}$ signal.

The high positive correlation of an increase in the density of phase slips $P(t)$ and the intensity of ionospheric irregularities $A(t)$ during geomagnetic disturbances as detected in this study points to the fact that the increase in slips
$P(t)$ is caused by the scattering of the GPS signal from ionospheric irregularities.

Nevertheless, the presence of the daytime maximum in the diurnal density distribution of fatal slips and of the TEC variation amplitude is inconsistent with existing data on the night-time intensity maximum of equatorial scintillations of transionospheric signals (Aarons, 1982; Yeh and Liu, 1982; Aarons et al., 1996, 1997; Klobuchar, 1997; Pi et al., 1997; Aarons and Lin, 1999). Besides, the level of slips at mid-latitudes was found to be unusually high. This would suggest that the reason for the increase in density of fatal phase slips during geomagnetic disturbances is that the type of GPS signal scattering from ionospheric irregularities is different from that causing signal scintillations in the case of the scattering from $F$ region irregularities at the local night-time in the equatorial zone.

7) During magnetic storms, the border between «mid» and «higher» latitudes moves equatorwards which brings scintillation producing ionospheric irregularities into the GPS to ground receiver rays. Phase slips of the GPS signal can be caused by the scattering from the geomagnetic field-aligned ionospheric $E$-layer small-scale irregularities (smaller than or on the order of the radius of the Fresnel zone), the amplitude of which increases with increasing geomagnetic disturbance level (Foster and Tetenbaum, 1991). Such a scattering can lead to strong signal fadings, and even to a particle screening of the «GPS satellite-receiver» propagation path.

8) The increase in density of fatal slips during geomagnetic disturbances on the dayside can also be caused by limitations of the design and adjustment of the receivers used in the analysis, rather than the GPS signal scattering from ionospheric irregularities. An increase in slip density can be caused in this case by an inadequate response of GPS receivers of some types (such as AOA Turbo Rogue) to fast changes in daytime TEC at the auxiliary frequency $L_{2}$. This effect prevents the identification of slips caused by phase fluctuations induced by the scattering from ion density irregularities. Unfortunately, we are unaware of any consistent investigation of this 
kind or published data lending support to such a point of view.

9) As an alternative, we developed and tested a new method for determining TEC variations using only data on the pseudo-range and phase measurements at fundamental frequency $L_{1}$. The standard deviation of the TEC variations obtained in phase measurements at two frequencies, $L_{1}-L_{2}$, and at fundamental frequency $L_{1}$, does not exceed 0.1 TECU, which permits this method to be used in strong disturbance conditions when phase slips at auxiliary frequency $L_{2}$ are observed.

Of course, phase measurements are more sensitive to equipment failures and to interferences in the GPS «satellite-receiver» channel when compared with group delay measurements which are directly used in solving navigation problems. Therefore, it is necessary to monitor the errors of determining the position of stationary sites of the global GPS network, based on the data in the RINEX-format available from the Internet, and to analyze these series in conjunction with the data on the conditions of the near-terrestrial space environment.

We are aware that this study has revealed only the key averaged patterns of this influence, and we hope that it would give impetus to a wide variety of more detailed investigations.

\section{Acknowledgements}

The authors are grateful to V.G. Eselevich, V.V. Yevstafiev, K.S. Palamarchouk, and G.V. Popov for their encouraging interest in this study and active participations in discussions. We are also indebted to S.A. Nechaev for the data from magnetic observatory Irkutsk made available to us. Thanks are also due to V.G. Mikhalkovsky for his assistance in preparing the English version of the $\mathrm{T}_{\mathrm{E}} \mathrm{X}$-manuscript. We greatly appreciate the Referee's effort to improve the submitted manuscript. This work was done with support from both the Russian foundation for Basic Research (grants 99-0564753 and 00-05-72026) and RFBR grant of leading scientific schools of the Russian Federation 00-15-98509.

\section{REFERENCES}

AARONS, J. (1982): Global morphology of ionospheric scintillations, Proc. IEEE, 70 (4), 360-378.

AARONS, J. and B. LIN (1999): Development of high latitude phase fluctuations during the January 10, April 10-11, and May 15, 1997 magnetic storms, J. Atmos. Terr. Phys., 61, 309-327.

AARons, J., M. MENDillo, E. KudEKI and R. YAntoscA (1996): GPS phase fluctuations in the equatorial region during the MISETA 1994 campaign, J. Geophys. Res., 101, 26,851-26,862.

AARons, J., M. MENDILlo and R. YANTOSCA (1997): GPS phase fluctuations in the equatorial region during sunspot minimum, Radio Sci., 32, 1535-1550.

AFRAIMOVICH, E.L. (2000): GPS global detection of the ionospheric response to solar flares, Radio Sci., 35, 1417-1424.

Afraimovich, E.L., K.S. Palamartchouk, N.P. PereValova and V.V. Chernukhov (1998a): Ionospheric effects of the solar eclipse of March 9, 1997, as deduced from GPS data, Geophys. Res. Lett., 25, 465-468.

Afraimovich, E.L., K.S. Palamartchouk and N.P. PEREVALOVA (1998b): GPS radio interferometry of travelling ionospheric disturbances, J. Atmos. Terr. Phys., 60, 1205-1223.

AFRAIMOVICH, E.L., E.A. Kosogorov, L.A. LEONOVICH, K.S. Palamarchouk, N.P. Perevalova and O.M. PIROG (2000a): Determining parameters of large-scale traveling ionospheric disturbances of auroral origin using GPS-arrays, J. Atmos. Terr. Phys., 62, 553-565.

Afraimovich, E.L., E.A. Kosogorov, K.S. PalaMARChOUK, N.P. PEREVAlova and A.V. Plotnikov (2000b): The use of GPS arrays in detecting the ionospheric response during rocket launchings, Earth, Planets, Space, 52, 1061-1066.

Afraimovich, E.L., N.P. Perevalova, A.V. Plotnikov and A.M. URALOV (2001a): The shock-acoustic waves generated by the earthquakes, Ann. Geophys., 19, 395-409.

AFrAimovich, E.L., E.A. KoSOGOROV, O.S. LESYUTA, I.I. USHAKOV and A.F. YAKOVETS (2001b): Geomagnetic control of the spectrum of traveling ionospheric disturbances based on data from a global GPS network, Ann. Geophys., 19, 723-731.

Afraimovich, E.L., A.F. Altyntsev, E.A. Kosogorov, N.S. LARINA and L.A. LEONOVICH (2001c): Ionospheric effects of the solar flares of September 23, 1998 and July 29, 1999 as deduced from global GPS network data, J. Atmos. Terr. Phys., 63, 1841-1849.

Basu, Santimay, E. MacKenzie and Sunanda Basu (1988): Ionospheric constraints on VHF/HUF communications links during solar maximum and minimum periods, Radio Sci., 23, 363-378.

Bhattacharrya, A., T.L. Beach, S. BAsu and P.M. KINTNER (2000): Nighttime equatorial ionosphere: GPS scintillations and differential carrier phase fluctuations, Radio Sci., 35, 209-224.

DAVIES, K. and G.K. HARTMANN (1997): Studying the ionosphere with the Global Positioning System, Radio 
Sci., 32, 1695-1703.

EVANS, J.V. (1977): Satellite beacon contributions to studies of the structure of the ionosphere, Rev. Geophys. Space Phys., 15, 325-350.

Foster, J.C. and D. TETENBAUM (1991): High resolution backscatter power observations of $440 \mathrm{MHz} E$-region coheren echoes in Millstone-Hill, J. Geophys. Res., 96, 1251-1261.

GURTNER, W. (1993): RINEX: The Receiver Independent Exchange Format Version 2, http://igscb.jpl.nasa.gov/ igscb/data/format/rinex 2.txt.

Ho, C.M., B.A. IIJIMA, X.P. LINDQWISTER, A.J.MANNUCCI, L. SPARKS, M.J. REYeS and B.D. WiLSON (1998): Ionospheric total electron content perturbations monitored by the GPS global network during two northern hemisphere winter storms, J. Geophys. Res., 103, 26,409-26,420.

Hofmann-Wellenhof, B., H. LichtenegGeR and J. Collins (1992): Global Positioning System: Theory and Practice (Springer-Verlag Wien, New York), pp. 327.

INTERFACE CONTROL DOCUMENT: ICD-200c; http:// www.navcen.uscg.mil/pubs/gps/icd200/.
Kharisov, V.N., A.I. PEROV and V.A. BOLDIN (1998): The Global Satellite Radio Navigation GLONASS System, (IPRZhR, Moscow), pp. 400 (in Russian).

KLOBUCHAR, J.A. (1986): Ionospheric time-delay algorithm for single-frequency GPS users, IEEE Trans. Aerosp. Electron. Syst., 23 (3), 325-331.

KLOBUCHAR, J.A. (1997): Real-time ionospheric science: the new reality, Radio Sci., 32, 1943-1952.

LANGLEY, R.B. (1998): GPS for Geodesy (Springer - Berlin, Heidelberg, New York, Barcelona, Budapest, Hong Kong, London, Milan, Paris, Singapore, Tokyo), 111-149.

PI, X., A.J. ManNuCCI, U.J. LindGWister and C.M. Ho (1997): Monitoring of global ionospheric irregularities using the worldwide GPS network, J. Geophys. Res. Lett., 24, 2283-2286.

SKONE, S. and M. DE JONG (2000): The impact of geomagnetic substorms on GPS receiver performance, Earth, Planets, Space, 52, 1067-1071.

SPOELSTRA, T.A. TH. and H. KeLDER (1984): Effects produced by the ionosphere on radio interferometry, Radio Sci., 19, 779-788.

YEH, K.C. and C.H. LIU (1982): Radio wave scintillations in the ionosphere, Proc. IEEE, 70 (4), 324-360. 